\title{
THE ACTION OF AN ALGEBRAIC TORUS ON THE AFFINE PLANE
}

\author{
BY \\ A. GUTWIRTH(1)
}

1. Introduction. Let $G$ be a connected algebraic group, $V$ a variety. $G$ is said to operate regularly on $V$ if we are given an everywhere defined rational map $g \times v \rightarrow g(v)$ of $G \times V \rightarrow V$ such that

$$
\begin{aligned}
g_{1}\left(g_{2}(v)\right) & =g_{1} g_{2}(v) \text { for any } g_{1}, g_{2} \in G, v \in V, \\
e(v) & =v \quad \text { for any } \quad v \in V .
\end{aligned}
$$

Denote by $k$ an algebraically closed field, by $A^{2}$ the affine plane over $k$, by $G_{m}$ the multiplicative group of the universal domain.

Our purpose in this paper is to study by elementary means the regular operation of $G_{m}$ on $A^{2}$. We shall denote by $\sigma$ a regular operation of $G_{m}$ on $A^{2}$ which is not the identity on $A^{2}, \sigma: G_{m} \times A^{2} \rightarrow A^{2}$, and by $\sigma(t)$ the restriction of this map given by $\sigma(t): t \times A^{2} \rightarrow A^{2}$, where $t \in G_{m}$.

We recall that an algebraic torus is the direct product of a finite number of multiplicative groups.

2. Change of coordinates in $A^{2}$. Let $(x, y)$ be a system of coordinates for $A^{2}$. Then if

$$
\left\{\begin{array} { l } 
{ x ^ { \prime } = f ( x , y ) , } \\
{ y ^ { \prime } = g ( x , y ) , }
\end{array} \quad \text { and } \quad \left\{\begin{array}{l}
x=f^{\prime}\left(x^{\prime}, y^{\prime}\right), \\
y=g^{\prime}\left(x^{\prime}, y^{\prime}\right),
\end{array}\right.\right.
$$

where $f, g \in k[x, y], f^{\prime}, g^{\prime} \in k\left[x^{\prime}, y^{\prime}\right]$, the system $\left(x^{\prime}, y^{\prime}\right)$ will also be an allowable system of coordinates for $A^{2}$. (Considered in one coordinate system the map $(x, y) \rightarrow\left(x^{\prime}, y^{\prime}\right)$ is an entire Cremona-transformation.)

3. Semi-invariant polynomials. Let a regular operation of $G_{m}$ on $A^{2}$ be given by $\sigma(t):(t, x, y) \rightarrow\left(x^{*}, y^{*}\right)$ where $t \in G_{m},(x, y)$ is a coordinate system for $A^{2}$, and $x^{*}, y^{*} \in k\left[x, y, t, t^{-1}\right]$.

If $f \in k[x, y]$; we define $\lambda_{t} f$, the $t$-translate of $f$, by

$$
\lambda_{t} f(x, y)=f\left(x^{*}, y^{*}\right) .
$$

We say that $f$ is a semi-invariant polynomial (abbreviated in the sequel as s.i.p.) of weight $s$ if $\lambda_{t} f=t^{s} f$, where $s$ is an integer. If $s=0, f$ is said to be an invariant polynomial.

Received by the editors November 16, 1961 .

(1) This work was supported by the NSF, under contract No. NSFG 9657. 
LEMMA 1. Let $f \in k[x, y]$ and suppose that $\lambda_{t} f=p f$, with $p \in k\left[x, y, t, t^{-1}\right]$. Then $p=t^{s}, s$ an integer.

Proof. Indeed $\lambda_{t-1}\left(\lambda_{t} f\right)=f$. Therefore $p\left(x, y, t^{-1}, t\right) \lambda_{t-1} p\left(x, y, t, t^{-1}\right)=1$, so that $p$ is independent of $x$ and $y$, and the result follows.

LEMMA 2. (a) Any factor of an s.i.p. is an s.i.p.

(b) Every semi-invariant rational function is the quotient of two s.i.p.

Proof. (a) Let $f_{1}, f_{2}, \cdots, f_{n}$ be the irreducible factors of a semi-invariant polynomial; then $\lambda_{t}\left(f_{1} \cdots f_{n}\right)=\lambda_{t} f_{1} \lambda_{t} f_{2} \cdots \lambda_{t} f_{n}=t^{s} f_{1} \cdots f_{n}$.

Therefore

$$
\lambda_{t} f_{i}=p\left(t, t^{-1}\right) \prod_{j \in I} f_{j}
$$

where $I$ is a subset of $1,2, \cdots, n, p\left(t, t^{-1}\right) \in k\left[t, t^{-1}\right]$. Taking $t=1$ in (3.2) it follows that $\lambda_{t} f_{i}=p\left(t, t^{-1}\right) f_{i}$.

(b) Let $\lambda_{t}(f / g)=t^{\alpha} f / g$; then $\left(\lambda_{t} f\right) g=t^{\alpha} f\left(\lambda_{t} g\right)$. Since $f$ and $g$ are relatively prime, the result follows by a standard divisibility argument.

COROLlaRY A. All polynomials of $k[x, y]$ which are semi-invariant under $\sigma$ are products of irreducible s.i.p.

Proposition 1. Every polynomial of $k[x, y]$ is a sum of s.i.p.

Proof. Let $f \in k[x, y]$, since $\lambda_{t} f \in k\left[x, y, t, t^{-1}\right]$ we have

$$
\lambda_{t} f=t^{\alpha}\left(f_{0}+t f_{1}+\ldots+t^{n} f_{n}\right)=t^{\alpha} \sum_{i=0}^{n} t^{i} f_{i}
$$

where, for all $i, f_{i} \in k[x, y]$ and $n \geqq 0$.

Since $\sigma$ is a regular operation of $G_{m}$ on $A^{2}$, the following conditions are satisfied:

(1) $\sigma(1)$ is the identity on $A^{2}$,

(2) $\sigma\left(t t_{1}\right)=\sigma(t) \sigma\left(t_{1}\right)$.

From (3.4) it follows that

$$
\left(t t_{1}\right)^{\alpha} \sum_{i=0}^{n} t^{i} t_{1}^{i} f_{i}=t_{1}^{\alpha} \sum_{i=0}^{n} t_{1}^{i}\left(\lambda f_{i}\right)
$$

Identifying corresponding coefficients of $t_{1}^{i+\alpha}$ in the two members of (3.5) we get

$$
\lambda_{t} f_{i}=t^{\alpha+i} f_{i}
$$

Therefore all the $f_{i}, i=1, \cdots, n$, in (3.5) are s.i.p. To finish the proof, take $t=1$ in (3.3).

Corollary B. Among the irreducible s.i.p. there are certainly two polynomials with independent linear terms. 
Proof. Apply Proposition 1 and Corollary A to $x, y \in k[x, y]$.

4. Invariant functions. Among the rational functions on $A^{2}$ some are invariant under $\sigma$. In the next few lemmas we investigate the connexions between those functions and the s.i.p.

LEMMA 3. The field of rational functions on $A^{2}$ which are invariant under $\sigma$ is a simple extension of $k$.

Proof. Consider the variety $W$ of orbits (see [3]) corresponding to the operation of $G_{m}$ on $A^{2} . W$ is of dimension one. Its function-field $k(W)$ is by Lüroth's theorem (see [4]) a simple extension of $k$. On the other hand $k(W)$ is exactly the subfield of $k(x, y)$ containing those functions of $k(x, y)$ which are invariant under $\sigma$. Q.E.D.

If $\sigma$ is such that all s.i.p. are of positive weight we have $k(W)=k(f / g)$, where $f$ and $g$ are s.i.p. of the same weight (see [2, p. 84]). In the sequel $k(W)$ will denote the subfield of $k(x, y)$ formed by the functions on $A^{2}$ invariant under $\sigma$.

Lemma 4. Let $\sigma$ be such that all s.i.p. are of positive weight and let $f / g$, ( $f$ and $g$ relatively prime) be a generator for $k(W)$. Consider an element $p=f+\mu g$, $\mu \in k$. Then all the irreducible factors of $p$ are equal.

Proof. If $f / g$ is a generator for $k(W)$, then so is $f+\mu g / g$. Therefore it suffices to prove that all the irreducible factors of $f$ are equal. Suppose that $f=f_{1}^{\alpha} f_{2}$, where $f_{1}$ is irreducible and $f_{2}$ contains no factors equal to $f_{1}$. Since the weights of $f, g, f_{1}$ are all positive $f_{1}^{a} g^{-b}$ will be an invariant function for some well chosen $a$ and $b$. Then $f_{1}^{a} g^{-b} \in k\left(f_{1}^{\alpha} f_{2} g^{-1}\right)$ i.e., since $k$ is algebraically closed

$$
\frac{f_{1}^{a}}{g^{b}}=\frac{c \prod_{i=1}^{n}\left(\left(f_{1}^{\alpha} f_{2} / g\right)+a_{i}\right)}{\prod_{j=1}^{m}\left(\left(f_{1}^{\alpha} f_{2} / g\right)+b_{j}\right)} \text { with } c, a_{i}, b_{j} \in k
$$

or

$$
f_{1}^{a} \prod_{j=1}^{m}\left(f_{1}^{\alpha} f_{2}+b_{j} g\right)=c g^{\varepsilon} \prod_{i=1}^{n}\left(f_{1}^{\alpha} f_{2}+a_{i} g\right),
$$

(if $\varepsilon$ is negative, we make the obvious change needed in order to get a polynomial identity).

Now $f_{1}^{\alpha}$ divides the right-hand member of (4.1). Since $f_{1} \Varangle g$ and $f_{1} \Varangle f_{2}$, it follows by standard divisibility arguments that $f_{2}=1$.

LEMMA 5. Let a regular operation of $G_{m}$ on $A^{2}$ be such that all s.i.p. are of positive weight. Let $p_{1}, p_{2}$ and $p_{3}$ be three irreducible s.i.p. of weights $\omega_{1}, \omega_{2}$ and $\omega_{3}$ respectively, such that $p_{1}, p_{2}, p_{3} \notin k$. 
Then they satisfy a relation of the type

$$
c_{1} p_{1}^{\alpha}+c_{2} p_{2}^{\beta}=p_{3}^{\gamma}
$$

where $\alpha \omega_{1}=\beta \omega_{2},(\alpha, \beta)=1 ; c_{1}, c_{2} \in k$.

Proof. Let $d$ be the g.c.d. of $\omega_{1}$ and $\omega_{2}$. Then $\omega_{1}=\beta d$ and $\omega_{2}=\alpha d$. Consider $\phi=p_{1}^{\alpha} p_{2}^{(-\beta)} \cdot \phi$ is invariant under $\sigma$, so that, if $f g^{-1}$ is a generator of $k(W)$ we have $p_{1}^{\alpha} p_{2}^{(-\beta)} \in k\left(f g^{-1}\right)$. Therefore

or

$$
p_{1}^{\alpha} p_{2}^{(-\beta)}=\frac{c \prod_{i=1}^{m}\left(f / g-a_{i}\right)}{\prod_{j=1}^{n}\left(f / g-b_{j}\right)} \text { with } c, a_{i}, b_{j} \in k
$$

$$
p_{1}^{\alpha} \prod_{j=1}^{n}\left(f-b_{j} g\right)=c p_{2}^{\beta} g^{\varepsilon} \prod_{i=1}^{m}\left(f-a_{i} g\right),
$$

(if $\varepsilon$ is negative, we multiply both members of (4.3) by $g^{-8}$, in order to get a polynomial identity).

Using Lemma 4 we then have, by standard divisibility arguments,

$$
p_{1}^{\alpha}=d_{1} f+d_{2} g, p_{2}^{\beta}=d_{3} f+d_{4} g, \quad d_{1}, d_{2}, d_{3}, d_{4} \in k,
$$

whence we can choose as generator for $k(W)$ the element $p_{1}^{\alpha} p_{2}^{(-\beta)}$. We now repeat this argument with the invariant function $p_{2}^{\omega_{3}} p_{3}^{\left(-\omega_{2}\right)}$, taking $p_{1}^{\alpha} p_{2}^{(-\beta)}$ as a generator for $k(W)$. The relation which we then get instead of (4.4) is

$$
p_{3}^{\gamma}=c_{1} p_{1}^{\alpha}+c_{2} p_{2}^{\beta}, \quad c_{1}, c_{2} \in k,
$$

where $\gamma$ is another positive number.

5. Invariant polynomials. Among the functions invariant under $\sigma$ there may, or may not, be polynomials. The two following lemmas treat both possibilities, and lead up to the main theorem.

LEMMA 6. If there exist nonconstant polynomials invariant under $\sigma$ there is at most one irreducible s.i.p. of positive weight and similarly at most one irreducible s.i.p. of negative weight.

Proof. Let $f$ be an invariant polynomial of the lowest degree, $f \notin k$, and let $k(W)=k(g / h)$, where $g$ and $h$ are relatively prime polynomials. Then

$$
f=\frac{c \prod_{i=1}^{n}\left(g / h-a_{i}\right)}{\prod_{j=1}^{m}\left(g / h-b_{j}\right)}=\frac{c h^{e} \prod_{i=1}^{n}\left(g-a_{i} h\right)}{\prod_{j=1}^{m}\left(g-b_{j} h\right)} \text { with } c, a_{i}, b_{j} \in k
$$

for some suitable $\varepsilon$. It follows that some $g-b_{j} h$ is a constant, and therefore that $k(W)=k(h)=k(f)$. 
Suppose now that $p_{1}$ and $p_{2}$ are different irreducible s.i.p. of positive weight. Then for an appropriate choice of $a$ and $b, a>0, b>0, \phi=p_{1}^{a} p_{2}^{(-b)}$ is invariant under $\sigma$. Therefore $p_{1}^{a} p_{2}^{-b} \in k\left(f_{i}\right)$. We then get a polynomial identity

$$
p_{1}^{a} \prod_{j=1}^{m}\left(f-c_{j}\right)=d p_{2}^{b} \prod_{i=1}^{m}\left(f-d_{i}\right) \quad \text { with } d, c_{j}, d_{i} \in k .
$$

As $p_{1}$ and $p_{2}$ are both irreducible it then follows that $p_{1}^{a} \in k(f), p_{2}^{-b} \in k(f)$, which 1s a contradiction. The proof is similar for $p_{1}$ and $p_{2}$ both of negative weight.

LEMMA 7. Let $\sigma$ be such that there are no nonconstant invariant polynomials. Then after a suitable change of coordinates (a translation) all s.i.p. will be polynomials without constant terms.

Proof. If there are no invariant polynomials the s.i.p. are either all of positive or all of negative weight. It is no loss of generality to take all weights positive.

Let $\sigma(t)$ be given by

$$
\begin{aligned}
& x^{*}=t^{\alpha}\left[f_{0}+t f_{1}+\cdots+t^{n} f_{n}\right], \\
& y^{*}=t^{\beta}\left[g_{0}+t g_{1}+\cdots+t^{m} g_{m}\right] .
\end{aligned}
$$

By (3.6) $f_{i}$ is an s.i.p. of weight $(\alpha+i)$. Since, by assumption, all s.i.p. are of positive weight we may suppose $\alpha=\beta=0$ in (5.1). On the other hand $f_{0}$ and $g_{0}$ are then reduced to constants, since there are no invariant polynomials. Hence (5.1) becomes

$$
\begin{aligned}
& x^{*}=a+t f_{1}(x, y)+\cdots+t^{n} f_{n}(x, y), \\
& y^{*}=b+t g_{1}(x, y)+\cdots+t^{m} g_{m}(x, y),
\end{aligned}
$$

where $m>0, n>0, a, b \in k$.

After the change of coordinates

we get

$$
X=x-a, \quad Y=y-b,
$$

$$
\begin{aligned}
& X^{*}=t f_{1}^{*}(X, Y)+\cdots+t^{n} f_{n}^{*}(X, Y), \\
& Y^{*}=t g_{1}^{*}(X, Y)+\cdots+t^{m} g_{m}^{*}(X, Y),
\end{aligned}
$$

where $f_{i}^{*}(X, Y)=f_{i}(X+a, Y+b), g_{j}^{*}(X, Y)=g_{j}(X+a, Y+b)$. In this new system of coordinates no s.i.p. of positive weight can have a constant term, since all powers of $t$ in the right-hand members of (5.4) are positive.

Proposition 2. Let $\sigma$ be a regular operation of $G_{m}$ on $A^{2}$. Then the set of all polynomials of $k[x, y]$ semi-invariant under $\sigma$ is generated over $k$ by two of its elements.

Proof. We distinguish two cases: 
(A) There are no invariant polynomials. Then all weights may be taken positive. By Lemma 7 we may suppose that all s.i.p. have no constant terms. We know (by Corollary B) that among the s.i.p. there are at least two $p_{1}$ and $p_{2}$, with independent linear terms. Since all s.i.p. have no constant term, $p_{1}$ and $p_{2}$ are irreducible. Denote by $\omega_{1}$, and $\omega_{2}$ the weights of $p_{1}$ and $p_{2}$ respectively. Let $p_{3}$ of weight $\omega_{3}$ be any other irreducible s.i.p. By Lemma 5 there exists a relation of the type:

$$
p_{3}^{\gamma}=c_{1} \mathrm{p}_{1}^{\alpha}+c_{2} p_{2}^{\beta}, \quad c_{1}, c_{2}, \in k,
$$

where $\alpha d=\omega_{2}, \beta d=\omega_{1},(\alpha, \beta)=1$. Then $\gamma \omega_{3}=\alpha \beta d$. Let $\alpha \leqq \beta$. The polynomials $p_{1}$ and $p_{2}$ have independent linear terms-so that $p_{3}^{\gamma}$ will have among its terms of lowest degree a term of the type $c_{3} x^{\delta} y^{\varepsilon}$ with $\delta+\varepsilon=\alpha, c_{3} \in k$. Therefore $\alpha=\mu \gamma$. Hence $\omega_{3}=\mu \beta d=\mu \omega_{1}$. Applying Lemma 5 again, this time to $p_{3}, p_{1}$ and $p_{2}$, we get

$$
c_{5} p_{3}+c_{6} p_{1}^{\mu}=p_{2}^{\varepsilon}, \quad c_{5}, c_{6} \in k .
$$

So that $p_{3}$ is a polynomial in $p_{1}$ and $p_{2}$.

(B) There exist invariant polynomials. By Lemma 6 there are at most two irreducible s.i.p., one of negative and one of positive weight. To conclude the proof it remains to show that they generate over $k$ all the invariant polynomials. Let $p_{1}$ and $p_{2}$ be these two irreducible polynomials of weights $\omega_{1}$ and $\omega_{2}$ respectively, $\omega_{1}>0, \omega_{2}<0$. Let $f$ be a generator for $k(W)$. Then $p_{1}^{\left(-\omega_{2}\right)} p_{2}^{\omega_{1}} \in k(f)$. The desired result follows by a divisibility argument, using the same methods as in Lemmas 4 and 5.

\section{Main theorems.}

Proposition 3. Any regular operation of $G_{m}$ on $A^{2}$ can, after a suitable change of coordinates, be reduced to the form

$$
(t, x, y,) \rightarrow\left(t^{\mu} x, t^{v} y\right)
$$

with $\mu$ and $v$ integers.

Proof. Let $\sigma$ be given by $(t, x, y) \rightarrow\left(x^{*}, y^{*}\right)$ where

$$
\begin{aligned}
& x^{*}=t^{\alpha} \sum_{i=0}^{n} f_{i} t^{i}, \\
& y^{*}=t^{\beta} \sum_{j=0}^{m} g_{j} t^{j},
\end{aligned}
$$

with $m \geqq 0, n \geqq 0, f_{i}, g_{j} \in k[x, y]$.

By Proposition 1 the $f_{i}$ and $g_{j}$ are s.i.p. By Proposition 2 all s.i.p. are polynomials in two irreducible s.i.p., say $X$ and $Y$. Then $f_{i}, g_{j} \in k[X, Y]$ and $X, Y \in k[x, y]$. 
On the other hand $x=\Sigma f_{i}, y=\Sigma g_{j}$, so that $x$ and $y$ are polynomials in $X$ and $Y$, say $x=P(X, Y), y=Q(X, Y)$. Therefore we have the relations

$$
\left\{\begin{array} { l } 
{ x = P ( X , Y ) , } \\
{ y = Q ( X , Y ) , }
\end{array} \quad \left\{\begin{array}{l}
X=X(x, y) \\
Y=Y(x, y)
\end{array}\right.\right.
$$

which define an allowable change of coordinates in $A^{2}$. In terms of the $(X, Y)$ coordinate system $\sigma$ is then given by

$$
(t, X, Y) \rightarrow\left(t^{\mu} X, t^{v} Y\right)
$$

since $X$ and $Y$ are s.i.p.

Proposition 4. If $G_{m}$ operates regularly on $A^{2}$ there is always a fixed point.

Proof. This is an obvious corollary of the previous proposition. Note that $G_{m}$ can operate on $A^{2}$ in such a way that there exists a curve of fixed points, but that in this case there is a fixed point adherent to every orbit.

7. Algebraic torus. The next two propositions concern the action of an algebraic torus on $A^{2}$.

Proposition 5. If an algebraic torus operates regularly on $A^{2}$ there always exists a fixed point.

Proof. Consider the algebraic torus $G_{m}^{(1)} \times G_{m}^{(2)}$ and let $\sigma_{1}$ and $\sigma_{2}$ respectively be the regular operations of $G_{m}^{(1)}$ and $G_{m}^{(2)}$ on $A^{2}$. Since $G_{m}^{(1)}$ and $G_{m}^{(2)}$ commute, the set of orbits corresponding to $\sigma_{1}$ is globally invariant under $\sigma_{2}$, and so is the set of fixed points of $\sigma_{1}$. Therefore, if $\sigma_{i}$ has only one fixed point, it is also a fixed point for $\sigma_{j}, i \neq j$. If on the other hand both $\sigma_{1}$ and $\sigma_{2}$ have curves of fixed points, the curve of fixed points of $\sigma_{1}$ is either a curve of fixed points for $\sigma_{2}$, or an orbit of $\sigma_{2}$. In either case it has at least one fixed point. This proves that $\sigma_{1}$ and $\sigma_{2}$ have a common fixed point. The proposition then follows by induction.

Proposition 6. Let an algebraic torus $G_{m}^{(1)} \times G_{m}^{(2)} \times \cdots \times G_{m}^{(r)}$ operate regularly on $A^{2}$. Then after an appropriate change of coordinates this operation can be described by

$$
\left(s_{1}, s_{2}, \cdots, s_{r}, x, y\right) \rightarrow\left(x \prod_{i=1}^{r} s_{i}^{\alpha_{t}}, y \prod_{j=1}^{r} s_{j}^{\beta_{j}}\right),
$$

where $s_{i} \in G_{m}^{(i)}, i=1,2, \cdots, l$, and the $\alpha_{i}$ and $\beta_{j}$ are integers.

Proof. Let a regular operation of $G_{m} \times G_{m}$ on $A^{2}$ be given by

$$
(s, t, x, y) \rightarrow\left(x^{*}, y^{*}\right)
$$

with $x^{*}, y^{*} \in k\left[x, y, s, s^{-1}, t, t^{-1}\right]$.

In particular (7.2) defines by restriction two regular operations of $G_{m}$ on $A^{2}$ which we denote by

$$
\sigma_{1}:(s, x, y) \rightarrow\left(x_{1}, y_{1}\right)
$$




$$
\sigma_{2}:(t, x, y) \rightarrow\left(x_{2}, y_{2}\right) \text {. }
$$

Suppose that the coordinate system is chosen so that $\sigma_{2}$ is of the form

$$
\sigma_{2}:(t, x, y) \rightarrow\left(t^{\alpha} x, t^{\beta} y\right) .
$$

Obviously in some allowable coordinate system $\sigma_{1}$ is of the form

$$
\left(s, x^{\prime}, y^{\prime}\right) \rightarrow\left(s^{\gamma} x^{\prime}, s^{\delta} y^{\prime}\right)
$$

and in this coordinate system the orbits have as equation

$$
x^{\prime \delta}+\mu y^{\prime \gamma}=0
$$

if we suppose that $\gamma \delta>0$. Let the change of coordinates from $\left(x^{\prime}, y^{\prime}\right)$ to $(x, y)$ be given by

$$
x^{\prime}=f(x, y), y^{\prime}=g(x, y), \quad f, g \in k[x, y] .
$$

Then in the $(x, y)$-coordinate system the equation of the orbits (7.7) is

$$
f^{\delta}+\mu g^{\gamma}=0 .
$$

These orbits have to be globally invariant under $\sigma_{2}$, so that $f^{\delta} / g^{\gamma}$ is a semi-invariant function for $\sigma_{2}$. By Lemma $2, f^{\delta}$ and $g^{\gamma}$ are then s.i.p., which means that both $f$ and $g$ are s.i.p. for $\sigma_{2}$, that is, in the $\left(x^{\prime}, y^{\prime}\right)$-coordinate system $x^{\prime}$ and $y^{\prime}$ are s.i.p. for $\sigma_{2}$. This proves that in the $\left(x^{\prime}, y^{\prime}\right)$-coordinate system both $\sigma_{1}$ and $\sigma_{2}$ are reduced to the canonical form of Proposition 3. The proof is similar if $\gamma \delta \leqq 0$. Our proposition follows by induction.

\section{REFERENCES}

1. A. Borel, Seminar on transformation groups, Annals of Mathematics Studies, No. 46, Princeton Univ. Press, Princeton, N.J., 1960.

2. C. Chevalley, Théorie des groupes de Lie. II, Hermann, Paris, 1951.

3. M. Rosenlicht, Some basic theorems on algebraic groups, Amer. J. Math. 78 (1956), 401-443.

4. P. Samuel, Some remarks on Lüroth's theorem, Mem. Coll. Sci. Univ. Kyoto Ser. A 27 (1953), 223-224.

TECHNION, HAIFA, ISRAEL 